\title{
Fatal progressive right heart failure in a pancreatic cancer patient
}

\author{
Jeong Tae Byoun, Jae Young Cho
}

Department of Cardiology, Wonkwang University Hospital, Iksan, Korea

Received: July 28, 2019

Revised: August 28, 2019

Accepted: September 10, 2019

Corresponding author:

Jae Young Cho

Department of Cardiology,

Wonkwang University Hospital, 895

Muwang-ro, Iksan 54538, Korea

Tel: +82-63-859-2512

Fax: +82-63-852-8480

E-mail: librato46@gmail.com
Pulmonary tumor thrombotic microangiopathy (PTTM) is a rare but fatal complication of cancer and causes pulmonary hypertension and acute/subacute right heart failure. PTTM is most commonly associated with gastric cancer and more rarely associated with pancreatic cancer. We report a case of progressive right heart failure associated with clinically diagnosed pancreatic cancer, suggesting PTTM.

Keywords: Pancreatic cancer; Pulmonary hypertension; Right sided heart failure; Thrombotic microangiopathy

\section{Introduction}

Pulmonary tumor thrombotic microangiopathy (PTTM) is a rare but fatal complication of cancer [1]. PTTM appears to be caused by unexplained pulmonary hypertension $(\mathrm{PH})$ and acute/subacute right heart failure. PTTM is histo-pathologically diagnosed by pulmonary vascular tumor microembolic nests with activated coagulation and obliterative intimal proliferation [2]. Almost all cases of PTTM, die within a few days or weeks of the onset of dyspnea. Hence, until recently, antemortem diagnosis has been very difficult [3]. In this study, we report a case of clinically diagnosed PTTM complicated with pancreatic cancer.

\section{Case}

This study was approved by the Institutional Review Board of Wonkwang University Hospital (IRB No: 2020-03-041).

A 72-year-old female was referred to our hospital with unresolved dyspnea. She had a history of hypertension, diabetes, asth- ma, and cerebral infarction. Two weeks before admission, dyspnea and cough developed. She was admitted to the local hospital for 10 days and was on antibiotics, including a third generation cephalosporin (ceftriaxone) and carbapenem (meropenem), resulting from suspected pneumonia.

At admission, she had resting dyspnea. Her body temperature was $37.1^{\circ} \mathrm{C}$, heart rate 108 beats $/ \mathrm{min}$, blood pressure $140 / 80$ $\mathrm{mmHg}$, respiratory rate 24 breaths/min, and oxygen saturation $84 \%$ while receiving oxygen via a nasal cannula at a rate of $5 \mathrm{~L} /$ min. Physical examination was notable for crackles without wheezing in the lungs and pitting edema in both lower legs. Arterial blood gas analysis showed $\mathrm{pH} 7.33, \mathrm{PCO}_{2} 52 \mathrm{mmHg}, \mathrm{PO}_{2} 56$ $\mathrm{mmHg}, \mathrm{HCO}_{3}^{-} 20.6 \mathrm{mM} / \mathrm{L}$, and $\mathrm{O}_{2}$ saturation $84.6 \%$. Laboratory tests revealed the following values: white blood cell count 8,840 / $\mu \mathrm{L}$, hemoglobin $11.2 \mathrm{~g} / \mu \mathrm{L}$, platelet count $393 \mathrm{~K} / \mu \mathrm{L}$, total bilirubin $0.17 \mathrm{mg} / \mathrm{dL}$, aspartate aminotransferase $20 \mathrm{IU} / \mathrm{L}$, alanine aminotransferase $14 \mathrm{IU} / \mathrm{L}$, alkaline phosphatase $97 \mathrm{IU} / \mathrm{L}$ (range, 30-120 IU/L), gamma glutamyl transferase 46 IU/L (range, 5-39 IU/L), blood urea nitrogen $29.9 \mathrm{mg} / \mathrm{dL}$, creatinine $0.69 \mathrm{mg} / \mathrm{dL}$,

Copyright(C) 2020 Yeungnam University College of Medicine

This is an Open Access article distributed under the terms of the Creative Commons Attribution Non-Commercial License (http://creativecommons.org/licenses/by-nc/4.0/) which permits unrestricted non-commercial use, distribution, and reproduction in any medium, provided the original work is properly cited. 
C-reactive protein $27.57 \mathrm{mg} / \mathrm{L}$ (range, $0-5 \mathrm{mg} / \mathrm{L}$ ), activated partial thrombin time $25.8 \mathrm{sec}$ (range, 23-35 sec), and international normalized ratio 1.15 . The level of brain natriuretic peptide and D-dimer were $1,921 \mathrm{pg} / \mathrm{mL}$ (range, $0-150 \mathrm{pg} / \mathrm{mL}$ ) and $5.8 \mu \mathrm{g} /$ $\mathrm{mL}$ (range, $0-0.5 \mu \mathrm{g} / \mathrm{mL}$ ), respectively; other laboratory results were unremarkable. Electrocardiogram showed sinus tachycardia at a rate of 110 beats/min, T wave inversion in V1-4, poor R-wave progression, and rightward axis deviation, known as 'right ventricle (RV) strain.'

Transthoracic echocardiography (TTE) (Fig. 1) showed RV dilatation with reduced systolic function, moderate tricuspid valve regurgitation (proximal isovelocity surface area radius, $5.1 \mathrm{~mm}$; jet area, $7.5 \mathrm{~cm}^{2}$ ), left ventricle septal flattening during the systolic phase, inferior vena cava plethora, and moderate $\mathrm{PH}$ (pulmonary artery systolic pressure [PASP], $65.7 \mathrm{mmHg}$; mean pulmonary arterial pressure, $44 \mathrm{mmHg}$ ) without obvious atrial or ventricular septal defect. Left ventricular systolic function was preserved with an ejection fraction of $71 \%$. Contrast-enhanced chest computed tomography (CT) with pulmonary arteriography was performed. CT showed the presence of multiple small consolidative lesions in both lung fields without evidence of pulmonary thromboembolism. A pulmonary function test showed moderate obstructive lung defect with good response to bronchodilator (forced expiratory volume in $1 \mathrm{sec}\left[\mathrm{FEV}_{1}\right] /$ forced vital capacity $[\mathrm{FVC}] 78 \%$, pre- $\mathrm{FEV}_{1} 0.79 \mathrm{~L}, 43 \% \rightarrow$ post-FEV $10.99 \mathrm{~L}, 58 \%$ ). A $15 \%$ decrease in the diffusing capacity of the lung for carbon monoxide was noted.

Given these findings, right-sided heart failure originating from untreated asthma and acute decompensation caused by community-acquired pneumonia was suggested as the initial diagnosis. However, despite antibiotics and proper diuretic-based heart fail- ure therapy, her dyspnea gradually worsened. On the 12th hospital day (HD), oxygen was supplied using a high flow nasal cannula $\left(\mathrm{FiO}_{2}, 0.4\right.$; flow, $\left.40 \mathrm{~L} / \mathrm{min}\right)$, and oxygen saturation was maintained within the range of $90 \%-95 \%$.

On the 14th HD, contrast-enhanced whole-body CT and ventilation/perfusion lung scans were performed to determine the cause of clinical deterioration. Chest CT showed numerous centrilobular nodules with "tree-in-bud" sign and pleural- enhancing nodules (Fig. 2A). Abdominopelvic CT showed a 3-cm less enhancing mass with ill-defined margins located at the pancreatic head and neck region, in addition to upstream pancreatic duct dilatation (Fig. 2B). A ventilation lung scan showed no significant decreased activity while a perfusion lung scan revealed multifocal peripherally distributed small perfusion defects in the bilateral lung fields (Fig. 3). Moreover, serum carbohydrate antigen 19-9 level was elevated $77.9 \mathrm{U} / \mathrm{mL}$ (range, $0-35 \mathrm{U} / \mathrm{mL}$ ); therefore, an endoscopic ultrasonographic-guided biopsy was needed for histopathological confirmation of pancreatic cancer. However, it was impossible to obtain the biopsy due to the unbearable severe dyspnea and opposition of the patient's family. ${ }^{18} \mathrm{~F}$-2-fluoro-2-deoxy-D-glucose-positron emission tomography integrated with computed tomography (FDG-PET/CT) was performed for diagnosis and staging of pancreatic cancer after discussion with the Pancreatobiliary Department (Fig. 4). This test showed an intensive hypermetabolic mass at the pancreatic head and neck portion with multiple common hepatic, portocaval, aortocaval, hepatoduodenal, para-aortic, left common iliac, mediastinum, right lower paratracheal, and right interlobar lymph node metastases in addition to numerous tiny bilateral lung and both pleural metastases.

According to these clinical and radiologic findings, PTTM sec-
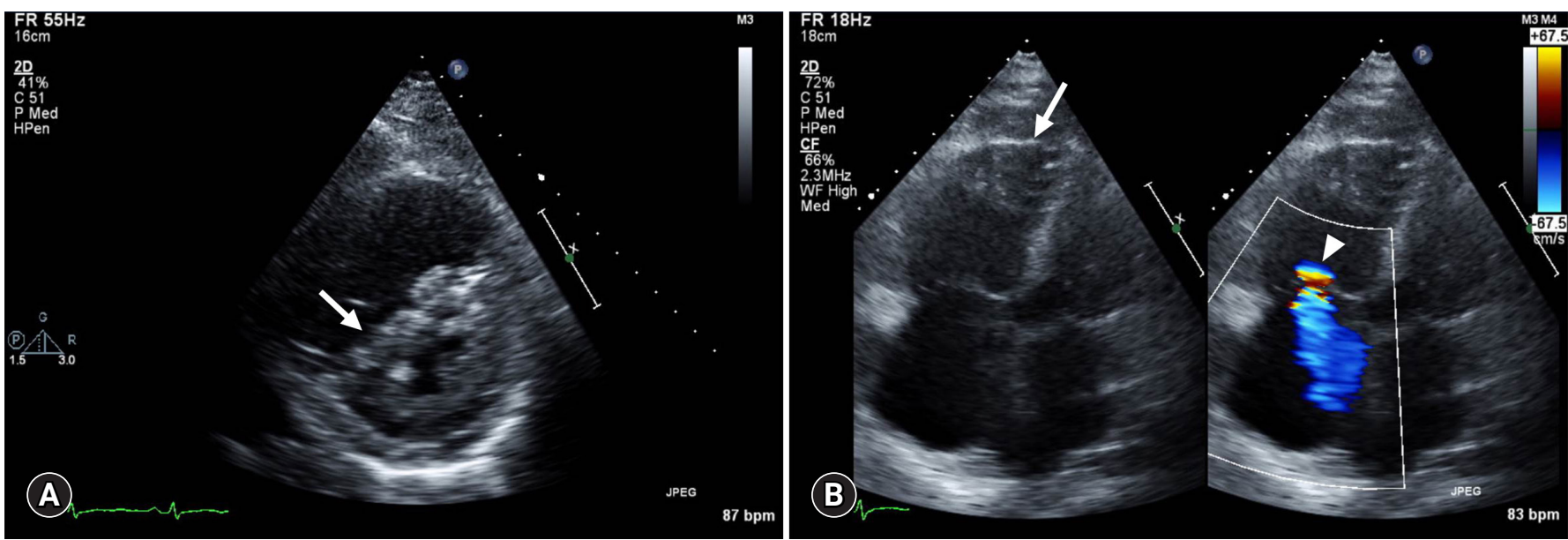

Fig. 1. Transthoracic echocardiography. (A) Parasternal short-axis view shows LV systolic septal flattening (arrow) with RV enlargement. (B) Apical 4 chamber view with color Doppler of the tricuspid valve shows RV enlargement (arrow) and moderate tricuspid regurgitation (arrowhead). LV, left ventricle; RV, right ventricle. 

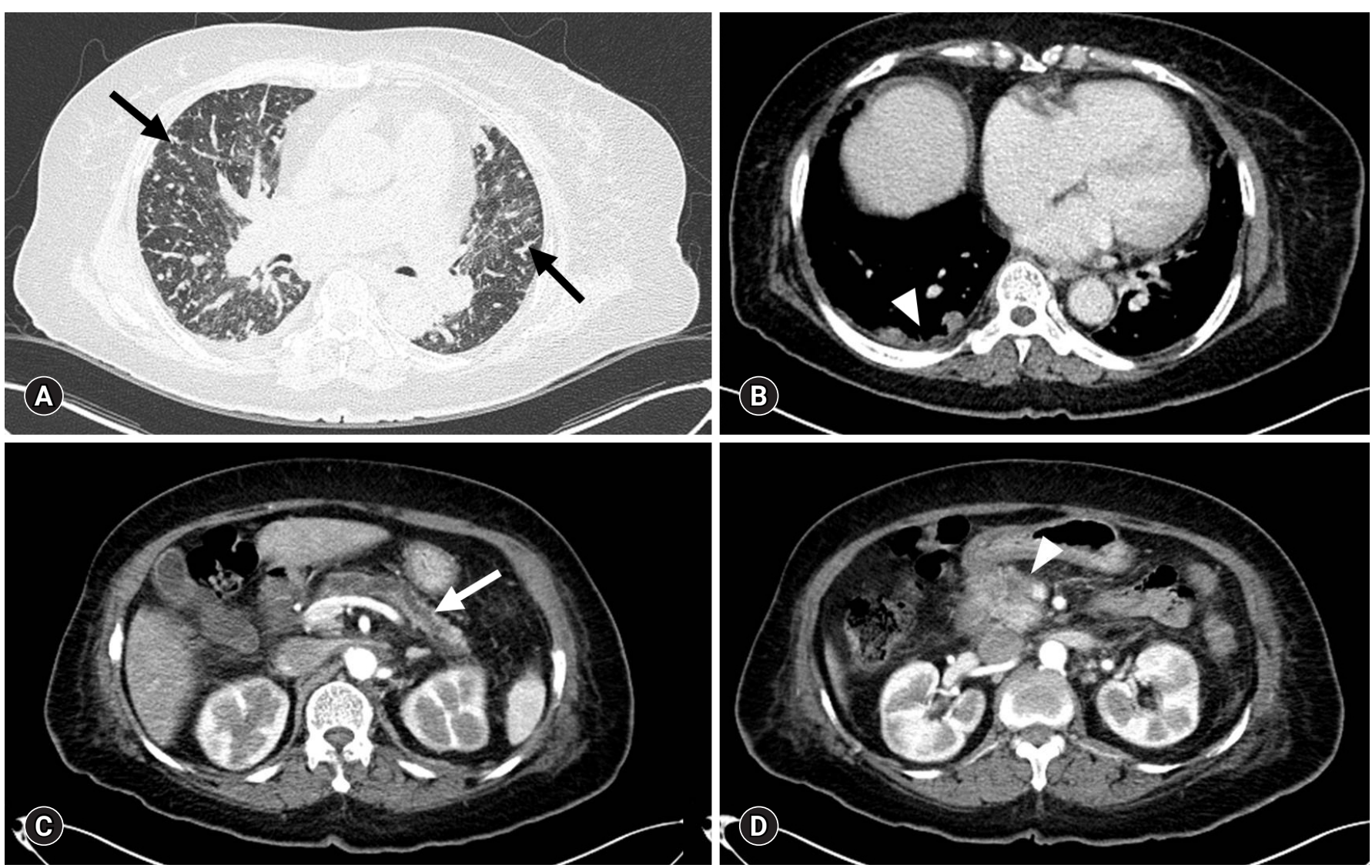

Fig. 2. Contrast-enhanced CT. (A) Chest CT (lung window) with centrilobular nodules with "tree-in-bud" sign (arrows). (B) Chest CT (mediastinal window) with pleural-enhancing nodules (arrowhead). (C) Abdominopelvic CT show upstream pancreatic duct dilatation (arrow). (D) Abdominopelvic CT with pancreatic head neoplasm (arrowhead). CT, computed tomography.

ondary to advanced pancreatic cancer was suggested as the final diagnosis. The clinical stage was TxN2M1, stage IV according to the 8th edition of the American Joint Committee on Cancer staging system [4]. Since the disease continuously progressed to multi-organ failure, including respiratory failure and acute kidney injury, only supportive care could be provided, and the patient died on the 27th day after diagnosis.

\section{Discussion}

PTTM, a rare complication associated with cancer, which can cause $\mathrm{PH}$ and right heart failure, was first described in 1990 by von Herbay et al. [1]. PTTM is histopathologically characterized by the presence of pulmonary vascular tumor microembolic nests with evidence of activation of the coagulation cascade, obstructive fibrointimal proliferation, and ultimately $\mathrm{PH}$ [2]. The most common primary malignancy associated with PTTM is gastric cancer, especially histologically mucinous, signet ring cells, and poorly differentiated subtypes [3]. It is known to be associated with a variety of carcinomas such as lung, breast, ovarian, and bladder can- cers, in that order $[5,6]$. However, cases with pancreatic cancer are relatively rare. Including our case, there have only been four reported cases associated with pancreatic cancer [6].

Clinical manifestations of PTTM are vague and may include a dry cough, dyspnea, and hypoxemia which are common symptoms in various cardiopulmonary diseases. Clinical suspicion is crucial because these can interfere with accurate diagnosis of PTTM. Most cases of PTTM were diagnosed postmortem with $\sim 79 \%$ of reported PTTM due to rapidly aggravating clinical course and fatal prognosis. PTTM tends to be under-diagnosed as most patients' families do not request an autopsy [7].

In laboratory tests, elevations of D-dimer, anemia, thrombocytopenia, and elevation of serum lactate dehydrogenase are the most common elevations but are non-specific [7]. In radiologic studies, centrilobular nodularity, ground glass opacity, and interlobular septal thickening have been reported in high resolution $\mathrm{CT}$ as in this case, but these findings may be seen in other lung diseases such as interstitial pneumonia [2]. On lung perfusion scans, perfusion defects distributed bilaterally may be observed unlike pulmonary thromboembolism which is limited by the vas- 


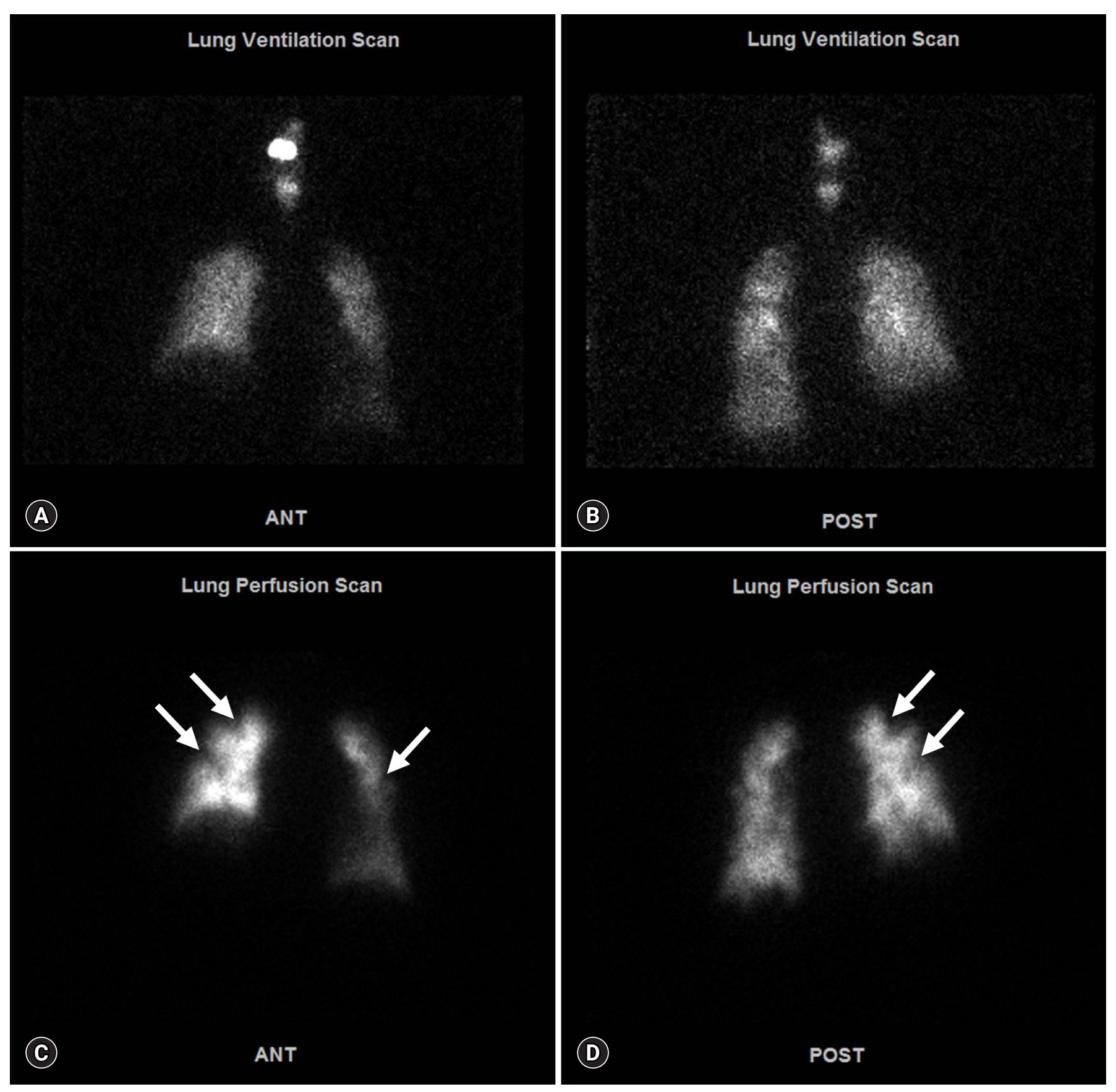

Fig. 3. Ventilation/perfusion lung scan. Anterior view (A) and posterior view (B) of ventilation lung scan present no significant decreased ventilation activity. Anterior view (C) and posterior view (D) of perfusion lung scan show multifocal peripherally distributed small perfusion defects in bilateral lung fields (arrows).

cular distribution. FDG-PET/CT can be effective for the diagnosis of primary malignancy. In PTTM, hypermetabolism of the area which can be confirmed as PTTM via biopsy was observed in FDG-PET/CT, but negative findings may be seen in small lesions or some subtypes such as the signet ring cell type [2]. TTE should be performed as soon as possible to determine the extent of $\mathrm{PH}$ and RV dysfunction; however, there is no evidence based on TTE to distinguish PTTM from other causes of PH. In the present case, the patient was also initially misdiagnosed with pneumonia accompanied by cor pulmonale which delayed PTTM diagnosis since there were no clinical indications of malignancy. Although contrast-enhanced whole-body CT and ventilation/perfusion lung scans were done almost concurrently and malignancy in addition to PTTM was diagnosed in a short inter- 

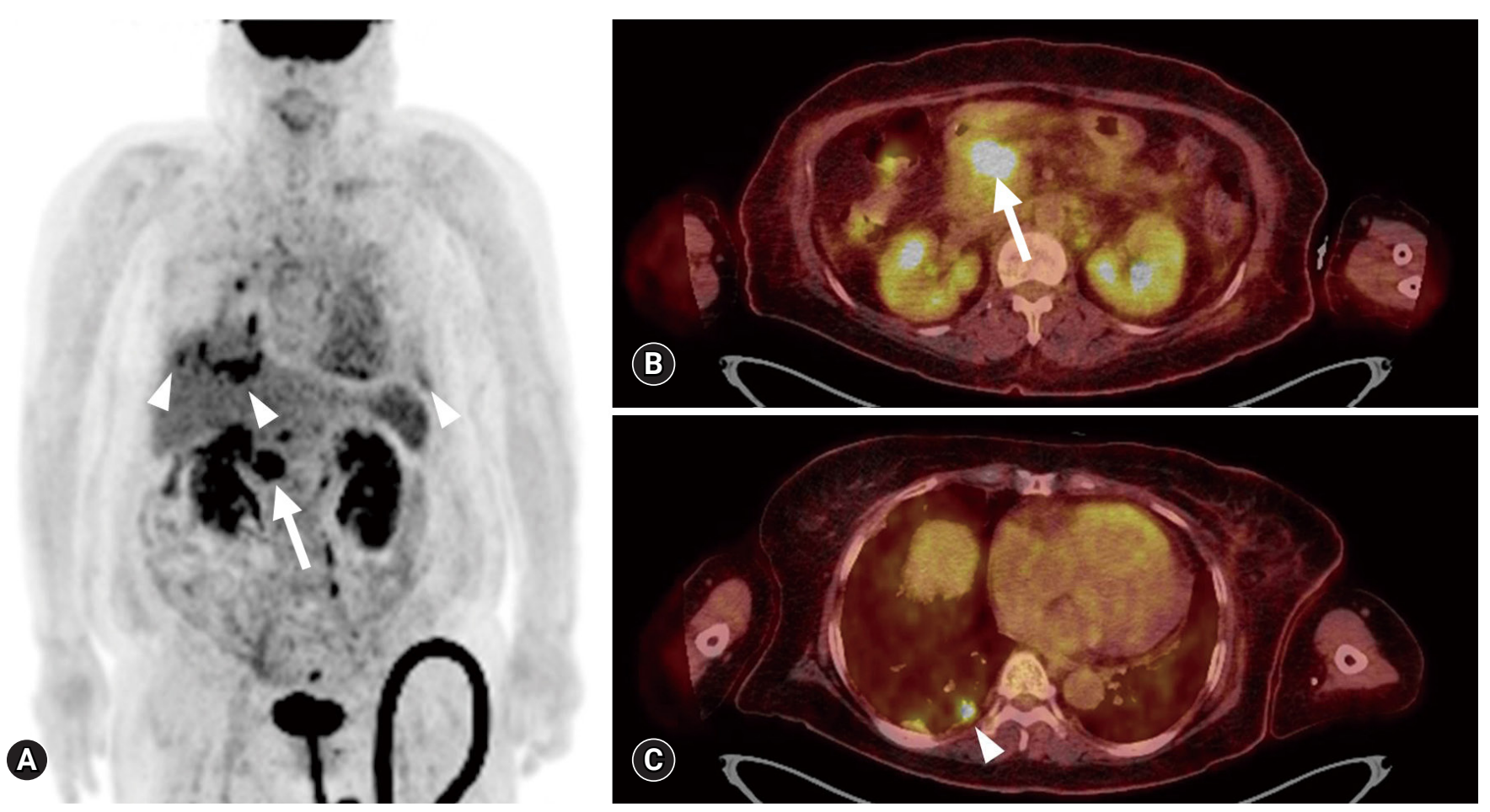

Fig. 4. ${ }^{18} \mathrm{~F}$-2-fluoro-2-deoxy-D-glucose-positron emission tomography integrated with computed tomography. Maximum intensity projection image $(A)$ and fusion axial views $(B, C)$ show a hypermetabolic mass at the pancreatic head and neck region (arrow) with systemic lymph nodes, bilateral lung and both pleural metastases (arrowhead).

val, this case identified the need for the thorough evaluation of $\mathrm{PH}$ including cancer. This is especially important if a patient with high PASP presents with progressive dyspnea. Prompt diagnosis of the systemic malignancy underlying PTTM should be done so at least it provides an opportunity for treatment with appropriate chemotherapy.

CT-guided lung biopsy, video-assisted thoracoscopic surgery, or bronchoscopy is necessary for histological confirmation of PTTM diagnosis because of the diagnostic limitations of these noninvasive tests. However, the risk of surgery or procedure is very high if accompanied by advanced $\mathrm{PH}$ and right heart failure. Alternatively, cytological examination of aspirated blood using a wedged pulmonary artery catheter with a sensitivity of $80 \%-88 \%$ and specificity of $82 \%-94 \%$ can be done. Additionally, if the TTE results are ambiguous, right heart catheterization may be useful for a more accurate assessment of $\mathrm{PH}[2,7]$.

To date, all cases reported as PTTM have died, and the average duration from symptom onset to death has been reported to be about 9.5 weeks. Furthermore, the mean duration of death after dyspnea was reported to be 16.2 days. Once the dyspnea occurs, the patient appears to experience rapid deterioration $[7,8]$. Various therapeutic methods including medical and oxygen therapy have been attempted, but no consensus has been established. It is thought that chemotherapy for primary malignancy reduces fibrointimal proliferation by reducing the number of cancer cells [2]. Thrombolysis is known to have no benefit. Anticoagulation appears to improve prognosis, but the evidence for its efficacy is weak [7]. There is no evidence that endothelin receptor antagonists, such as bosentan and ambrisentan, reduce pulmonary vascular remodeling but can be used to reduce pulmonary vasoconstriction [3]. Dexamethasone has been reported to improve the natural course in a few cases in combination with anticoagulation and chemotherapy [3]. Imatinib, a tyrosine kinase inhibitor that blocks the phosphorylation of the platelet-derived growth factor receptor, may be beneficial for survival. Bevacizumab, a vascular endothelial growth factor inhibitor, may be more effective when combined with imatinib than when used alone [2]. However, since most of these treatments are based on case reports, systematic research will be needed in the future.

In a patient presenting with progressive right heart failure of unknown origin, PTTM should be considered regardless of the presence or absence of known cancer upon high index of suspicion. Although prognosis remains very poor, prompt antemortem diagnosis of PTTM will allow specific and aggressive therapies, such as chemotherapy, to be used and may improve the prognosis in limited cases. 


\section{Acknowledgments}

\section{Conflicts of interest}

No potential conflict of interest relevant to this article was reported.

\section{Funding}

This study was supported by Wonkwang University in 2019.

\section{ORCID}

Jeong Tae Byoun, https://orcid.org/0000-0002-0407-2568

Jae Young Cho, https://orcid.org/0000-0001-7972-6223

\section{References}

1. von Herbay A, Illes A, Waldherr R, Otto HF. Pulmonary tumor thrombotic microangiopathy with pulmonary hypertension. Cancer 1990;66:587-92.

2. Price LC, Seckl MJ, Dorfmüller P, Wort SJ. Tumoral pulmonary hypertension. Eur Respir Rev 2019;28:pii: 180065.
3. Price LC, Wells AU, Wort SJ. Pulmonary tumour thrombotic microangiopathy. Curr Opin Pulm Med 2016;22:421-8.

4. Chun YS, Pawlik TM, Vauthey JN. 8th edition of the AJCC cancer staging manual: pancreas and hepatobiliary cancers. Ann Surg Oncol 2018;25:845-7.

5. Fujishiro T, Shuto K, Shiratori T, Kono T, Akutsu Y, Uesato M, et al. A case report of pulmonary tumor thrombotic microangiopathy (PTTM) caused by esophageal squamous cell carcinoma. Esophagus 2013;10:247-51.

6. Patrignani A, Purcaro A, Calcagnoli F, Mandolesi A, Bearzi I, Ciampani N. Pulmonary tumor thrombotic microangiopathy: the challenge of the antemortem diagnosis. J Cardiovasc Med (Hagerstown) 2014;15:828-33.

7. Godbole RH, Saggar R, Kamangar N. Pulmonary tumor thrombotic microangiopathy: a systematic review. Pulm Circ 2019;9: 2045894019851000.

8. Uruga H, Fujii T, Kurosaki A, Hanada S, Takaya H, Miyamoto A, et al. Pulmonary tumor thrombotic microangiopathy: a clinical analysis of 30 autopsy cases. Intern Med 2013;52:1317-23. 